\title{
Las Revistas Científicas Colombianas y la Política Pública de COLCIENCIAS
}

\section{The Colombian Scientific Journals and COLCIENCIAS' Public Policy}

\author{
ANDRÉS ESCOBAR-ESPINOZA ${ }^{1}$ \\ Universidad de Cartagena \\ aescobare@unicartagena.edu.co \\ MENIS MERCADO-MEJIA ${ }^{2}$ \\ Universidad de Cartagena \\ mmercado2@unicartagena.edu.co
}

La evaluación de revistas científicas del Índice Bibliográfico Nacional Publindex ha sido producto de un proceso de mejoramiento progresivo en miras a la consolidación de las publicaciones seriadas científicas en el ámbito nacional. Éste fue iniciado a finales de los años noventa, pero fue en 2001 cuando se establecen los primeros requisitos y condiciones necesarias que las publicaciones seriadas científicas colombianas debían cumplir. El Departamento Administrativo de Ciencia, Tecnología e Innovación (COLCIENCIAS) inicia en 2002 a través del mandato establecido en el decreto 1279, señalando cuatro categorías (A1, A2, B y C), estableciéndose de esta manera el Sistema Nacional de Indexación de Publicaciones Seriadas de Ciencia, Tecnología e Innovación conformado por la Base Bibliográfica Nacional (BBN), el Servicio de Indexación de Publicaciones Seriadas de CTel Nacionales y el Servicio de Homologación de Publicaciones Seriadas de CTel Extranjeras.

Es así como en 2002, existían 91 revistas científicas indexadas y categorizadas en el país, y 10 años después, se cuenta con 466 publicaciones indexadas como consecuencia de la apropiación de los procesos editoriales al interior de las distintas instituciones en el país (Publindex, 2013). Sin embargo, este crecimiento en la producción científica nacional estuvo acompañado de un bajo nivel de visibilidad e impacto en la comunidad científica internacional, y en ciertos niveles, con características endogámicas. Por lo que

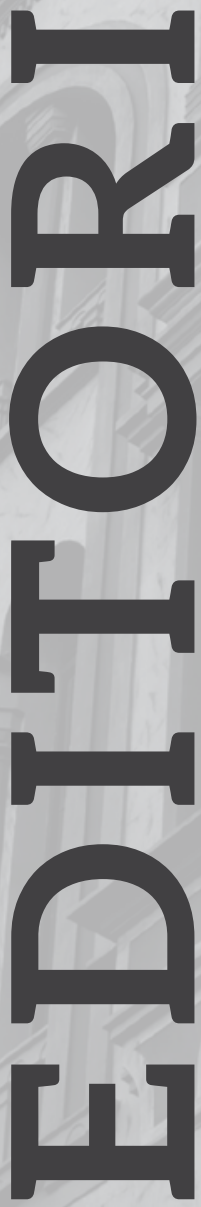


se establece la necesidad que las revistas científicas nacionales deben fortalecer sus procesos editoriales en función de los siguientes criterios: calidad científica, calidad editorial, estabilidad, visibilidad e impacto de las publicaciones (Publindex, 2013).

La gestión editorial determina la calidad científica de la publicación seriada en su conjunto, al establecer de manera clara, estandarizada y rigurosa los procesos de evaluación por pares idóneos, la originalidad de los documentos, con particular énfasis a los artículos de investigación e innovación. Lo anterior se logra mediante una organización y articulación del Consejo Editorial, Editor, y sus colaboradores (pares evaluadores).

Las normas editoriales específicas de la revista científica, articulada con los lineamientos editoriales institucionales, garantiza el cumplimiento de criterios editoriales estándares, además de que estos sean públicos y de fácil acceso. Alcanzando de esta manera, el cumplimiento de la periodicidad declarada públicamente, el cual se refiere a la estabilidad de la publicación seriada, que se constituye como un factor importante en la evaluación internacional de las mismas.

De la misma forma, la publicación científica seriada debe ser de fácil acceso y de libre circulación (Romero, 2013). Lo anterior implica que las publicaciones científicas no sean exclusivamente impresas, sino que preferiblemente, deben orientarse a contar con versiones electrónicas y facilitar su acceso en línea de sus contenidos a través de portales web o plataformas especializadas de gestión editorial. De esta manera, se promueve el acceso internacional a las mismas. Sin embargo, para promover la visibilidad internacional de las publica- ciones científicas seriadas a través de la integración en Sistemas de Indexación y Resumen (SIR), se necesita una gestión editorial eficiente que garantice al menos, la estabilidad, la calidad editorial y científica de la publicación seriada.

Con relación al impacto de las publicaciones científicas, el modelo propuesto se orienta a las métricas populares (Journal Citations Report - JCR, Scimago Journal Ranking - SJR) las cuales han sido desarrolladas por las principales multinacionales líderes del sector, y se refieren al análisis de las citaciones que reciben los artículos publicados en las revistas científicas evaluadas, las cuales son posteriormente categorizadas en función del área de conocimiento de cobertura declarada por la publicación.

Sin embargo, el modelo de evaluación propuesto no considera, para evaluar el impacto de las publicaciones científicas, métricas alternativas tales como la propuesta por Hirsch (2005) conocida popularmente como Índice $\mathrm{H}$ para individuos, a partir del cual proliferaron diversas versiones (Ruane \& Tol, 2008; Gagolewski \& Grzegorzewski, 2009) y sugirieron nuevos índices que permiten la medición del impacto y la productividad en el campo científico aplicable a diversas unidades de medida (Bras-Amoros et al., 2011; Silagadze, 2010).

Los nuevos lineamientos propuestos por COLCIENCIAS, exigen ajustes a las políticas editoriales de las instituciones con miras a fortalecer los procesos editoriales de sus publicaciones científicas seriadas y que éstas consigan un mejoramiento en su posicionamiento internacional a través de indicadores de calidad necesarios en el campo científico especializado y competitivo.

Lo anterior se constituye en un avance en 
busca de internacionalizar las publicaciones seriadas colombianas. Los resultados se verán reflejados en la medida que los equipos editoriales y las instituciones editoras trabajen coordinadamente para garantizar la inclusión de sus publicaciones en redes internacionales de conocimiento. Para el caso del Caribe colombiano, el posicionamiento de sus publicaciones científicas es una necesidad para la región, contribuyendo así a la reducción de las brechas existentes con las regiones más desarrolladas del país.

En éste sentido, la Revista Panorama Económico de la Universidad de Cartagena, se encuentra fortaleciendo sus procesos editoriales para garantizar la calidad científica de la misma, y con miras a incrementar su visibilidad, ya cuenta con la plataforma de gestión editorial Open Journal System (OJS), la cual permite tener acceso abierto disponible en línea a más de 220 artículos publicados desde su primer número, además de un control eficiente y adecuado de los documentos durante todo el proceso de gestión editorial. Por otro lado, y con una apropiada gestión, se proyecta su integración en los Sistemas de Indexación y Resumen (SIR), incluyendo también el cálculo de su impacto (mediante el análisis de citaciones). El objetivo de la Revista, es posicionar e incrementar su visibilidad en los principales índices nacionales e internacionales.

\section{REFERENCIAS BIBLIOGRÁFICAS}

Maria Bras-Amorós, Josep Domingo-Ferrer, Vicenç Torra (2011), "A bibliometric index based on the collaboration distance between cited and citing authors", Journal of Informetrics, 5 (2), 248-264. Disponible en: http:// dx.doi.org/10.1016/j.joi.2010.11.001.

Gagolewski, M. \& P. Grzgorzewski (2009), "A Geometric approach to the construction of scientific impact indices", Scientometrics, 81(3): 617-634. Disponible en: http://dx.doi. org/10.1007/s11192-008-2253-y

Hirsch, J. E. (2005), "An index to quantify an individual's scientific research output", Proceedings of the National Academy of Sciences USA, 102 (46), 16569-16572. Disponible en: http://dx.doi.org/10.1073/ pnas.0507655102

Romero, Z. (2013), "Hacia un nuevo modelo de medición que refleje las capacidades investigativas de los colombianos", Revista Saber, Ciencia y Libertad, 8(2), 13-16.

Ruane, F. \& R. S. Tol (2008), "Rational (successive) H-indices: An application to economics in the Republic of Ireland", Scientometrics, 75 (2), 395-495. Disponible en: http://dx.doi. org/10.1007/s11192-007-1869-7

Publindex (2013), Nuevo Modelo de Indexación de Publicaciones Seriadas de CTI-Publindex. Bogotá: Colciencias.

Silagadze, S.K. (2010). "Citation entropy and research impact estimation", Acta Physica Polonica Series B, 41, 2325-2333. 
